P093 DISTINCT AND OVERLAPPING ACTIVITIES OF IL-17A AND TNF ON THE EXPRESSION OF PROINFLAMMATORY CYTOKINES AND MMPS IN PSORIATIC ARTHRITIS: RATIONALE FOR ANTI-IL-17A/ANTI-TNFALPHA COMBINATION THERAPY?

${ }^{1} \mathrm{X} \mathrm{Xu}^{*},{ }^{1} \mathrm{~N}$ Davelaar, ${ }^{1} \mathrm{~A}-\mathrm{M}$ Otten-Mus, ${ }^{1} \mathrm{P}$ Asmawidjaja, ${ }^{1} \mathrm{~J}$ Hazes, ${ }^{2} \mathrm{D}$ Baeten, ${ }^{1} \mathrm{M}$ Vis, ${ }^{1} \mathrm{R}$ Bisoendial, ${ }^{1} \mathrm{E}$ Lubberts. ${ }^{1}$ Erasmus MC Rotterdam, Rotterdam; ${ }^{2}$ Academic medical centre, Amsterdam, Netherlands

10.1136/annrheumdis-2018-EWRR2018.109

Introduction TNF and IL-17A are proinflammatory cytokines critically involved in the pathogenesis of psoriatic arthritis (PsA). Currently, targeting TNF is the first choice of a biologic disease-modifying antirheumatic drug (bDMARD) in PsA. However, up to $30 \%$ of patients receiving anti-TNF monotherapy fail to respond and require switching to a second TNF-inhibitor or bDMARD with different modes of action. Strategies targeted at neutralising IL-17A have shown beneficial effects on skin, enthesitis, dactylitis and joint inflammation.

Objectives We explore the effect of neutralising IL-17A versus anti-TNF on the expression of proinflammatory cytokines and metalloproteinases (MMPs) and whether dual-therapy targeting TNF and IL-17A may have superior activity than treatment with either agent alone.

Methods An allogeneic co-culture system was used comprising synovial fluid $\mathrm{T}$ helper (Th) memory cells and synovial fibroblasts (SF), derived from patients with active PsA. Anti-CD3 and CD28 stimulation was used during culture, and anti-IL17A antibody (Secukinumab), anti-TNF $\alpha$ antibody (Adalimumab), or the combination were added. PsA unstimulated synovial Th memory cells co-cultured with PsA SF were included as a control group as well as an isotype antibody control group. After 72 hours, supernatants were harvested for ELISA and cells were lysed for qPCR analysis.

Results Anti-TNF Ab treatment had no effect on IL-17A levels and neutralisation of IL-17A did not influence the TNF production in co-culture system. Both anti-TNF and anti-IL-17A single treatment significantly inhibited the production of IL-8 and reduced the mRNA expression of IL-1 $\beta$. Interestingly, neutralising IL-17A resulted in a significant suppression of IL6 level which was not reduced by anti-TNF. Anti-TNF inhibited the production of MMP-3 and only the combination of anti-TNF and anti-IL-17A resulted in a significant suppression of MMP-1 levels and MMP-9 mRNA expression. MMP-13 mRNA expression was significantly suppressed by anti-TNF but not by anti-IL-17A. However, neutralising both showed a significant improvement in downregulating MMP-13 mRNA expression compared to single treatment. Moreover, anti-IL$17 \mathrm{~A}$ reduced RANK mRNA expression significantly compared to anti-TNF, but no additive effect was noted for combination blocking. Interestingly, only neutralising both IL-17A and TNF significantly reduced the mRNA expression of RANKL. OPG mRNA expression was not influenced by either treatment or both.

Conclusions Neutralising IL-17A or TNF in the PsA synovial $\mathrm{T}$ cell-SF co-culture system resulted in overlapping but also distinct effects on proinflammatory cytokine expression. TNF inhibition markedly suppress different MMPs with mostly an additional effect upon neutralisation of IL17A. Neutralisation both IL-17A and TNF is needed to downregulate RANKL expression which changes the RANKL/OPG balance. Together, these data suggest that dual therapy targeting IL-17A and
TNF may be superior in their activity to protect against erosive arthropathy in PsA than treatment with either agent alone.

Disclosure of interest X. Xu: None declared, N. Davelaar: None declared, A.-M. Otten-Mus: None declared, P. Asmawidjaja: None declared, J. Hazes: None declared, D. Baeten: None declared, M. Vis: None declared, R. Bisoendial: None declared, E. Lubberts Grant/research support from: Novartis

\section{P094 ASSESSMENT OF INTRACRANIAL VESSELS AND VASCULAR LESIONS IN RHEUMATOID ARTHRITIS. A DETAILED TRANSCRANIAL DOPPLER, CAROTID ULTRASOUND AND BRAIN MRI STUDY}

\begin{abstract}
${ }^{1} \mathrm{Z}$ Kardos, ${ }^{2} \mathrm{C}$ Oláh, ${ }^{3} \mathrm{M}$ Sepsi, ${ }^{4} \mathrm{~A}$ Sas, ${ }^{3} \mathrm{~L}$ Kostyál, ${ }^{5} \mathrm{H}$ Bhattoa, ${ }^{1} \mathrm{~K}$ Hodosi, ${ }^{1} \mathrm{~A}$ Domián, ${ }^{6} \mathrm{G}$ Kerekes, ${ }^{7} \mathrm{~L}$ Tamási, ${ }^{4} \mathrm{~A}$ Valikovics, ${ }^{8} \mathrm{D}$ Bereczki, ${ }^{1} \mathrm{Z}$ Szekanecz ${ }^{*}$. ${ }^{1}$ Department of Rheumatology; ${ }^{2}$ Department of Neurosurgery; ${ }^{3}$ Department of Radiology; ${ }^{4}$ Department of Neurology, Borsod County Teaching Hospital, Miskolc; ${ }^{5}$ Department of Laboratory Medicine; ${ }^{6}$ Department of Angiology, University of Debrecen, Faculty of Medicine; ${ }^{7}$ Department of Rheumatology, Borsod County Teaching Hospital, Debrecen; ${ }^{8}$ Department of Neurology, Semmelweis University, Budapest, Hungary
\end{abstract}

\subsection{6/annrheumdis-2018-EWRR2018.110}

Introduction Stroke has been associated with rheumatoid arthritis (RA). Vascular physiology should be assessed in the preclinical vascular state. Assessment of intracranial vessels includes transcranial doppler (TCD). TCD performance requires intact temporal acoustic windows (TAW). Failure of TAW (TAWF) is present in $8 \%-20 \%$ of people. This may be due to pathological bone metabolism.

Objectives We assessed RA patients and healthy controls by transcranial Doppler (TCD), carotid ultrasonography and brain MRI. We wished to determine preclinical pathophysiological changes in the cerebral vasculature.

Methods TAWF, temporal bone thickness and texture were determined by ultrasound and CT. BMD and T-scores of multiple bones were determined by DEXA. Several bone biomarkers were assessed by ELISA. Altogether 63 female RA patients and 60 age-matched controls underwent TCD assessment of the medium cerebral (MCA), basilar and vertebral arteries. Pulsatility(PI), resistance(RI) indices and circulatory reserve capacity (CRC) were determined. The presence of carotid plaques and intima-media thickness (cIMT) were also determined. Intracerebral vascular lesions were investigated by brain MRI. RA subsets include MTX- and biologic-treated patients.

Results In RA, the right and left TAW were undetectable in $35 \%$ and $53 \%$ of patients, respectively. In controls, TAWF was only present in $20 \%$ of subjects. Temporal bone was thicker, and texture was more heterogeneous in RA patients was controls $(p<0.05)$. These TAW features were generally more common on the left side. There was close association between TAWF, bone thickness and texture $(p<0.05)$. These TAW parameters all correlated with age, however TAW failure and texture also correlated with serum osteoprotegerin. TAW bone thickness inversely correlated with hip BMD $(\mathrm{p}<0.05)$.

Conclusions TAWF, thicker and heterogeneous temporal bones were associated with RA. These features have been associated with bone loss and OPG production. Bone loss seen in RA may result in OPG release and stimulation of bone formation around TAW. This may be the first study to show increased distal MCA and basilar artery occlusion in RA as determined by TCD. RA patients also exert CRC defect. We also 
confirmed increased carotid plaque formation, increased cIMT. Biologics may beneficially influence some parameters in the intracranial vessels.

Disclosure of interest None declared

\section{P095 PLASMA LEVELS OF HSP90 ARE INCREASED IN PATIENTS WITH SYSTEMIC SCLEROSIS WITH MORE SEVERE ORGAN INVOLVEMENT}

\begin{abstract}
1,2 H Storkanova*, 1,2S Oreska, ${ }^{2,3} \mathrm{M}$ Spiritovic, ${ }^{1,2} \mathrm{~K}$ Pavelka, ${ }^{1,2} \mathrm{~J}$ Vencovský, ${ }^{4} \mathrm{~J}$ Distler, 1,2 L Senolt, ${ }^{1,2} \mathrm{R}$ Becvar, ${ }^{1,2} \mathrm{M}$ Tomcik. ${ }^{1}$ Department of Rheumatology, 1st Faculty of Medicine, Charles University, Prague, Czech Republic; 'Institute of Rheumatology, Prague, Czech Republic; ${ }^{3}$ Department of Physiotherapy, Faculty of Physical Education and Sport, Charles University, Prague, Czech Republic; ${ }^{4}$ Department of Internal Medicine III, Institute for Clinical Immunology, University of Erlangen-Nuremberg, Erlangen, Germany
\end{abstract}

\subsection{6/annrheumdis-2018-EWRR2018.111}

Introduction Our previous study demonstrated that Hsp90 is overexpressed in the skin of patients with systemic sclerosis $(\mathrm{SSc})$, in cultured SSc fibroblasts and preclinical models of SSc. We showed that Hsp90 is a regulator of TGF- $\beta$ signalling and its inhibition prevents the stimulatory effects of TGF- $\beta$ on collagen synthesis and dermal fibrosis in three preclinical models of SSc. ${ }^{1}$

Objectives The aim of this study was to evaluate Hsp90 in the circulation of SSc patients and characterise its potential association with skin changes and SSc-related features.

Methods A total of 91 patients (78 females; mean age 52.7; disease duration 6.0 years; diffuse cutaneous (dc)SSc/limited cutaneous $\left.(\mathrm{lc}) S S_{c}=38 / 53\right)$ who met the ACR/EULAR 2013 criteria for SSc and 85 age-/sex- matched healthy controls (HC) were included. Plasma Hsp90 was measured by ELISA. Data are presented as median (IQR).

Results Plasma Hsp90 levels were increased in SSc patients

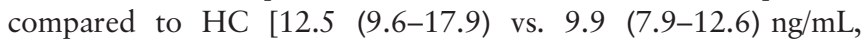
$\mathrm{p}=0.001$ ], but no difference between $\mathrm{lcSS} c$ and dcSSc were

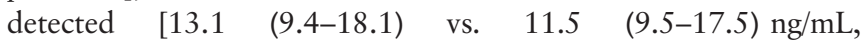
$\mathrm{p}=0.316$ ]. Hsp90 levels in all patients positively correlated with CRP $(\mathrm{r}=0.313, \mathrm{p}=0.006)$. Furthermore, Hsp90 was increased in patients with interstitial lung disease (ILD) compared to those without ILD [12.8 (10.2-17.9) vs. 10.3 (8.616.6) $\mathrm{ng} / \mathrm{mL}, \mathrm{p}=0.045]$ and was negatively associated with functional parameters of ILD: FVC $(=-0.299, \mathrm{p}=0.011)$, FEV1 $(r=-0.256, p=0.031)$, DLCO $(r=-0.303, p=0.009)$ and $\mathrm{SpO}_{2}(\mathrm{r}=-0.317, \mathrm{p}=0.038)$. In addition, only in patients with dcSSc, Hsp90 levels positively correlated with the mRSS $(r=0.437, p=0.006)$. Hsp90 concentrations were not significantly affected by other main clinical parameters of SSc.

Conclusions We demonstrated higher plasma levels of Hsp90 in SSc patients compared to healthy controls. Concentrations of extracellular Hsp90 increase with higher inflammatory activity, with deteriorated lung functions in ILD and also with the extent and severity of the skin involvement in patients with diffuse cutaneous SSc. These data further highlight the role of Hsp90 as a significant regulator of fibroblast activation and tissue fibrosis in SSc.

\section{REFERENCE}

1. Tomcik M, et al. Ann Rheum Dis 2014;73(6):1215-22.

Acknowledgements Supported by AZV - 16-33542A and SVV - 260373.

Disclosure of interest None declared

\section{P096 SYNOVIAL TISSUE REMODELLING AS A MEANS OF TISSUE MEMORY}

1,2 Olmos Calvo*, ${ }^{1} \mathrm{RA}$ Byrne, ${ }^{1} \mathrm{M}$ Bonelli, ${ }^{1} \mathrm{~B}$ Niederreiter, ${ }^{1} \mathrm{~F}$ Alasti, ${ }^{1} \mathrm{~T}$ Karonitsch, ${ }^{3} \mathrm{~J}$ Holinka, ${ }^{1} \mathrm{G}$ Steiner, ${ }^{1} \mathrm{JS}$ Smolen, ${ }^{2} \mathrm{P}$ Ertl, ${ }^{1} \mathrm{HP}$ Kiener. ${ }^{1}$ Rheumatology, Medical University of Vienna; ${ }^{2}$ Faculty of Technical Chemistry, Vienna University of Technology; ${ }^{3}$ Orthopedic Surgery, Medical University of Vienna

\subsection{6/annrheumdis-2018-EWRR2018.112}

Introduction The synovium demonstrates a distinct cellular structure with a densely packed synovial lining layer that sits on top of a loosely organised sublining layer. In rheumatoid arthritis (RA), this tissue hosts the inflammatory reaction and undergoes drastic structural changes. We hypothesise that this inflammatory remodelling results in tissue dysfunction, thereby perpetuating the inflammatory process. To explore the relationship between tissue structure and function, we use a 3D human synovial organoid culture system to model healthy and diseased synovium.

Methods FLS from RA patients were resuspended in Matrigel and cultured as 3D organoids for an extended period of time. This allows FLS to re-establish a synovial tissue-like structure. To mimic inflammation, 3D synovial organoids were challenged with TNF. A re-stimulation experiment was designed in which synovial organoids were exposed to TNF for 10 days, followed by a 3 day wash out phase. Thereafter, the organoids were re-stimulated with TNF. To prevent TNF-driven remodelling, Marimastat, a broad MMP inhibitor, was added during the first TNF stimulation. For selected experiments, synovial tissue-like structure was assessed by $\mathrm{HE}$ staining in paraffin embedded sections of synovial organoids. qPCR and RNA Seq were used in gene expression profiling. IL-6, IL-8 and MMP3 protein production was determined by ELISA.

Results TNF-stimulated synovial organoids demonstrated lining layer hyperplasia and cellular condensation in the sublining layer. After TNF-stimulation, gene expression of MMP1, MMP3 and IL-6 and protein production of IL-6, IL-8 and MMP3 was upregulated. Upon re-stimulation of synovial organoids with TNF, protein levels of IL-6, IL-8 and MMP3 were significantly increased when compared to previous stimulations. In order to explore whether this effect is due to TNFinduced structural changes, tissue remodelling was blocked using Marimastat. IL-6 protein level was reduced in 21\%. To further explore the increased TNF response upon re-stimulation, epigenetic mechanisms are currently being tested.

Conclusions TNF stimulation has a direct effetc in synovial organoids function and structure. TNF-driven tissue remodelling is associated with an increased in IL-6, IL-8 and MMP3 expression response upon re-stimulation. Inhibition of TNFinduced remodelling partially prevents this effect. Additional epigenetic mechanisms may account for tissue memory.

Disclosure of interest None declared

\section{P097 ANTI-TNF TREATMENT IMPROVES VASCULAR FUNCTION VIA SUPPRESSION OF GALECTIN-3 EXPRESSION DURING INFLAMMATORY ARTHRITIS}

K Sime*, E Hughes, EH Choy, AS Williams. Cardiff University, Cardiff, UK

\subsection{6/annrheumdis-2018-EWRR2018.113}

Introduction The link between rheumatoid arthritis (RA) and cardiovascular disease (CVD) is well established but not yet fully understood. Anti-TNF treatment, e.g. with Enbrel, is one 